\title{
The Credit Risk Pricing with Particle Filter Approach
}

\author{
Her-Jiun Sheu ${ }^{1}$ Chih-Liang Liu ${ }^{2}$ \\ ${ }^{1}$ Department of Management Science and Graduate Institute of Finance, National Chiao Tung University; No. 1001 \\ Ta Hsueh Rd., Hsinchu 300, Taiwan. Phone: +886 2 2349-4975; Fax: +886 2 2349-4976; e-mail: \\ hjsheu@faculty.nctu.edu.tw \\ ${ }^{2}$ Institute of Business and Management, National Chiao Tung University; No. 118, Sec. 1, Chung Hsiao W. Rd., \\ Chung Cheng District, Taipei 100, Taiwan. Phone: +886 2 2381-2386 ext. 57675; Fax: +886 2 2349-4976; e-mail: \\ chlliu.bm93g@nctu.edu.tw
}

\begin{abstract}
Traditional evaluation of firm's market value in credit risk analysis could be contaminated by market noises. The purpose of this article is to price the credit risk in the distance to default with particle filter approach. Compared to the traditional methods, the estimate of the distance to default could be evaluated precisely. Another finding is that the inflation of variation in firm's value caused by the market noises could be reduced.
\end{abstract}

Keywords: Particle Filter, Sequential Monte Carlo, Credit Risk, Distance to Default.

\section{Introduction}

The accurate and online measurement of credit risk could directly and critically affect the relative credit spread, market rating and credit derivatives pricing. Accuracy of default probability is thus crucial for researchers in the study of credit risk problems and their characteristics. On the other hand, the postanalysis of firm's credit evaluation, credit rating and other risk index, is not precise and efficient for market trading. The study of accuracy in default probability and online measurement turns out to be the main issue in credit risk analysis

According to Black-Scholes (1973), Merton (1974) derives the structural model to evaluate the risk structure. KMV extends the idea to measure the credit risk. The information with default content, whether or not affected by macroeconomic factors, individual financial stress or other shocks, could be reflected immediately in an efficient market. Traditional KMV model could be especially useful in efficient market, U.S. market alike. The market asset value and its volatility could be evaluated with the equity and debt market. But the distance to default derived by KMV may fail in the inefficient market with noises. Therefore the market asset value could not be directly measured with those latent variables. Another alternative is to measure the unobserved variables, market asset value and its volatility, with filtering techniques, including Kalman filter or Particle filter.

The filtering techniques are to track the latent variables with online and accurate advantages. Due to the nonlinearity and the disturbance of the distance to default model, the Kalman filter is no longer appropriate for measuring the credit risk. Particle filter provides a simulation based approach without complex derivation of closed form solution. The nonlinear and non-Gaussian properties make research more available in estimating financial market model.

In this article, we adopt particle filter approach in measuring the credit risk with distance to default. Due to the chaos in financial market, the evaluation of the firm's market asset value could be contaminated by the market noises. With particle filter technique, the estimate of the distance to default could be purely and properly evaluated respectively. Another finding in the article is that the variation of firm's market value could be reduced. The later part will contain the application and the problem of the structural model, the adjusted model with particle filter approach, the simulation results and the conclusions.

\section{Structural Model}

The determinants of credit risk include firm specific and macroeconomic factors, see Elton et al. (2001) and Bulter and Fauver (2005). Credit risk could be evaluated with several indices, such as credit rating, see Kisgen (2005), credit score, see Collin-Dufresne et al. (2001), credit spread, see Yu (2005), and distance of default, Vassalou and Xing (2004).

Black-Scholes (1973) derive the options pricing model (B-S model). Many B-S based pricing model extend the original approach and break several limitations. Those approaches are so called structural model. The latest B-S based model is Moodys' KMV. In the default risk model, traditional options pricing model calculate the distance of default (DD). The 
distance to default at time $\mathrm{t}$ can be defined as $D D_{t}=\left[\ln \left(V_{A, t} / X_{t}\right)+\left(\mu-\sigma_{A}^{2} / 2\right) t\right] / \sigma_{A} \sqrt{t}$

where $D D_{t}$ is the distance to default at time t, $V_{A, t}$ is the firm's market value at time t, $X_{t}$ is the debt value, $\mu$ and $\sigma_{A}{ }^{2}$ are the drift and the volatility of the firm's equity prices and $t$ is the maturity time.

This article provides a more stable and precise B$\mathrm{S}$ based credit risk model, distance to default with particle filter technique. The problem that the default probability is affected by some market interruptions still remains. The firm's market asset value can not be measured directly in inefficient market. The firm's asset market value could be properly evaluated using the sum of the debt value and the equity market value which could be measured by the multiplication of the stock price and number of shares outstanding. What makes the measure inefficient is that the observed stock prices can diverge from its real value due to the market noises. The one to one relationship between the unobserved asset value and the observed stock price is broken. The equity market value is determined by two main sources, the true stock price and the uncertain noises (Duan and Fulop 2005).

Another contaminated parameter is the asset volatility. The contaminated asset market value could also affect the market volatility. Ignoring the market noises could non-trivially inflate/underestimate the true asset volatility. The evaluation of the parameters, the asset market value and the market volatility, is a nonlinear estimation. Particle filter is a simulatedbased technique to generate sequential predictions for general nonlinear or non-Gaussian state space models.

\section{Pricing the Distance to Default with Particle Filter}

The application of Monte Carlo simulation in finance is introduced by Boyle (1977), especially in options pricing. After the random number is generated and the process has been calculated, the future price could be discounted by the risk free rate as an expected value of the current asset price. Kalman filter could be adopted to find the smoothed estimates in the trace problem. In the analysis of distance to default, the posterior density is no longer Gaussian at every time step. Another critical is that the state space system, based on options pricing model, is a nonlinear formula. Kalman filter may fail to properly estimate the non-linear systems, see Pitt and Shephard (1999).

Particle filter is a sequential Monte Carlo method based on resampling the point mass (or particle) in the probability densities, which could be applied to any state space model and which generalize the traditional linear Kalman filter. The main idea of particle filter is to represent the required probability density function as a set of random samples. If the number of samples is very large, it may effectively provide an equivalent representation of the required probability density function. Without knowing the specific function of the density, the properties of the density of the default could be understood from those samples.

\subsection{Filtering Techniques}

The estimation effectiveness of particle filter is determined by the weighting procedure. The data with more important information will be given higher weights and the weights given process could be seen as the filtering procedure. Therefore, the data with more information content will be kept crucially in the estimation. Two steps in the procedure are taken to implement the filtering approach. The likewise Bootstrap is carried out in the beginning to sample from the prior density. The sampled particles are given weights and then the weighted particles are re-sampled again to estimate the unknown posterior properties.

The required posterior density function could be represented by the random samples with particle filter procedure associated the importance weights. Whether or not the procedure can be successfully applied is determined by the Bootstrap alike weighting process. The weight is calculated by the prior densities as a function of the density conditioned on the particles divided by the sum of these intensities. Bayes' rule and importance sampling are recursively used to reweight the particles. The Bayesian estimation is to construct the probability density function of the state based on all the available information. It also implies that the particles are weighted higher if the particles contain more accurate information.

The observed equity prices could diverge from their equilibrium values due to microstructure noises (e.g. illiquidity, asymmetric information and price discreteness, etc.). The particle filter could be adopted to filter out irrelevant information. In the credit risk analysis, the default probability is a function of the stock price which contains some unrelated noises. Ignoring market noises could non-trivially inflate one's estimate of the true asset volatility. The filter procedure could be adopted to keep the market price with fewer irrelevant interruptions and thus the default probability could be estimated accurately.

The equity value could be derived from a one to one transformation of the firm's unobserved asset market value depending on some model parameters. Particle filter technique could be used to estimate the real firm value with stock prices (Duan and Fulop, 2005). The filter is a specific sampling procedure that is relative to the true asset value (Pitt and Shephard, 
1999). The relationship between the unobserved asset value and the observed equity prices predicted by the equity pricing formula is masked by trading noise.

Thus, the state space system could be modeled. The measurement vector represents the (noisy) observations that are related to the state vector: $\ln S_{t}=\ln S\left(V_{t} ; \sigma_{t}, X_{t}, r_{t}, t\right)+\delta v_{t}$ where $\ln S_{t}$ is the logarithmic equity prices, $V_{t}$ is the firm's unobserved market value, $\sigma_{t}$ is the latent variation of market value, $X_{t}$ is the debt value, $\delta$ is a multiplicative error structure for the noises, $v_{t}$ is iid standard normal random variables and $S(\cdot)$ is a nonlinear pricing formula. The transition equation (the state vector) contains all relevant information required to describe the system under investigation: $\ln V_{t+1}=\ln V_{t}+\left(\mu-\sigma_{t}^{2} / 2\right) h+\sigma \sqrt{h} \varepsilon_{t+1}$ where $\varepsilon_{t}$ is iid standard normal random variables. Gordon et al. (1993), Doucet et al. (2001), Arulampalam et al. (2002) and Lin et al. (2005) indicate that particle filter is a simulation based method to generate consecutive prediction and filter distributions for general state space models. It could be seen as a sequence of different points to represent the distributions of the unobserved state variables.

\subsection{The Sampling / Importance Re- sampling (SIR) Algorithms}

Sequential Monte Carlo is a recursive Bayesian filter by simulations. $V_{t}^{(m)}, m=1, \cdots, \mathrm{M}$ is a set of $\mathrm{M}$ particles and $f\left(V_{t} \mid \cdot\right)$ is the particle set with equal weights. $\Theta$ is the parameter set in the density. The prediction density is

$$
\widehat{f}\left(V_{t+1} \mid I_{t+1}, \Theta\right) \propto f\left(S_{t+1} \mid V_{t+1}, \Theta\right) \hat{f}\left(V_{t+1} \mid I_{t}, \Theta\right)
$$

where $I_{t}$ is the set of parameters determined as follows in the $D D$ model and the empirical filter density is

$$
\widehat{f}\left(V_{t+1} \mid I_{t}, \Theta\right) \propto \frac{1}{M} \sum_{m=1}^{M} f\left(V_{t+1} \mid V_{t}^{(m)}, \Theta\right)
$$

The purpose is to construct the posterior probability density function of the state based on all available information, including the set of the received measurements. The procedure of the Bootstrap alike approach can be processed as the following steps:

Step 1 Prediction Stage with Equal Weighted Particles

$$
\widehat{f}\left(V_{t+1} \mid I_{t}, \Theta\right) \rightarrow\left\{V_{t+1}^{(m)} ; m=1, \cdots, M\right\}
$$

Step 2 Filter Weights

$$
\pi_{t+1}^{(m)}=w_{t+1}^{(m)} / \sum_{k=1}^{M} w_{k=t+1}^{(m)} \text { where } w_{t+1}^{(m)}=f\left(S_{t+1} \mid V_{t+1}^{(m)}, \Theta\right)
$$

Step 3 Re-sampling and Updating

The variance of importance weights will not grow over time (stochastically)

$$
\left\{\left(V_{t+1}^{(m)}, \pi_{t+1}^{(m)}\right) ; m=1, \cdots, M\right\}
$$

The final procedure is to compute the default probability. The adjusted Merton model becomes:
$C\left(V_{t} ; \Theta\right)=\frac{-1}{t} \ln \left(\frac{V_{t}}{X_{t}} f\left(-d_{t}\right)+e^{-r t} f\left(d_{t}-\sigma_{t} \sqrt{t}\right)\right)-r_{t}$

and the adjusted default probability turns out to be $P\left(V_{t} ; \Theta\right)=f\left(\ln \left(X_{t} / V_{t}\right)-\left(\mu-\sigma_{t}^{2} / 2\right) t / \sigma_{t} \sqrt{t}\right)$

The parameters are set as follows: $t=251$ is the number of time steps, $M=50$ is the number of particles, $\mu=0.012$ is the drift in the process, $\sigma_{t}=0.15$ is the volatility in the process, $V_{0}=200$ is the initial state of firm value, $X_{t}=50$ is the value of debt (the exercise price in the original B-S model), $r=1$ is the trading noise variance and the initial variance of the states is 5 .

\section{Simulation Results}

In this article, there is no outlier in the simulation process and the auxiliary variable is not carried out in the next study. 50 particles are adopted in the simulated process, that is, there are 50 simulating paths in each experiment and each path contains 250 steps excluding the initial value.
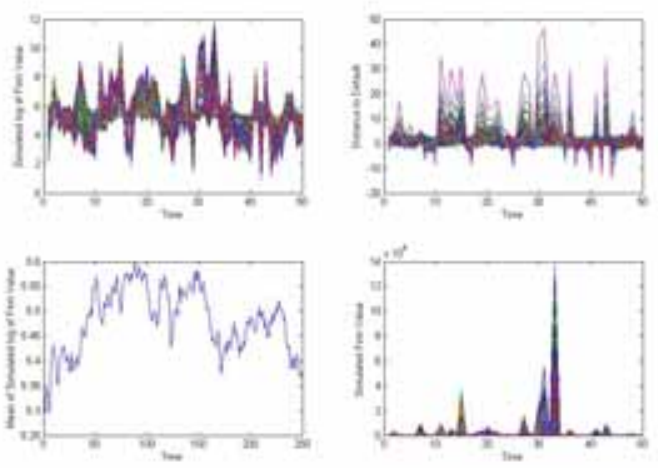

Fig. 1: Simulation of Firm's Value and DD.

Monte Carlo simulation of the firm's value and the distance to default is shown in figure 1. The distance to default can not be evaluated directly. There are enormous variations in distance to default. The second procedure is to weight the sampling. The data with more information would be given more weights. There are about half of the density with lower weights. Thus, we take the higher weighted data to the next procedure. The Bayesian estimates of the weight are shown in the figure 2 . The particles are not unevenly distributed and the weights are not quite equal. The filter approach could be applied in the next procedures.
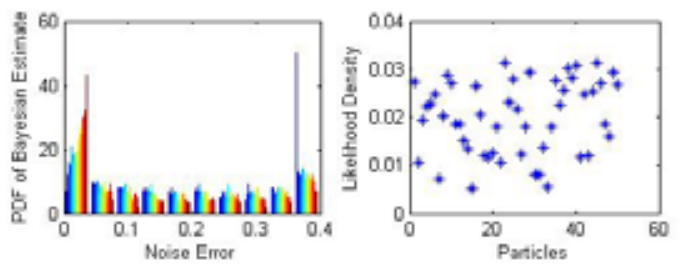

Fig. 2: The Bayesian Weights. 
After weighting the data with information content, the noises of the measurement process and the transition process could be calculated. The advanced estimate of the distance to default is relatively stable. The main reason is due to the reduction of the noise contaminations. As the simulation results, it also shows that particle filter is useful in eliminating the market noise in pricing the credit risk.
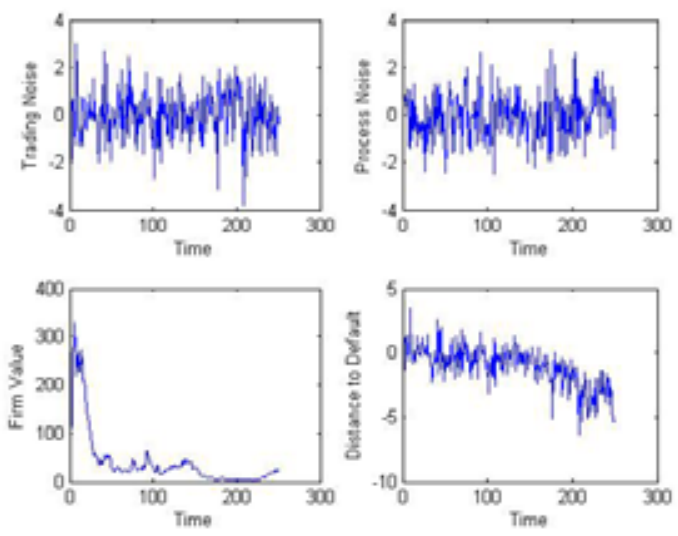

Fig. 3: Firm's Market Value and DD with Particle Filter.

Traditional evaluation of firm's market value in credit risk analysis could be easily contaminated by market noises. Those interruptions are irrational expectations. As the result, the firm's market value is hard to be evaluated directly. With the adoption of particle filter approach, the pure market firm value could be calculated via the density of weighted resamples with information content. The noises are also filtered out in the evaluation process. The remained data could be seen as the ones with fewer market noises. The estimated firm's value could be seen as a real market value and the relative distance of default pricing of individual firm could be correctly calculated.

\section{Conclusion}

In this study, the credit risk in distance to default is evaluated with particle filter approach. Due to the chaos in financial market, the evaluation of firm's market value could be contaminated by the market noises. Particle filter could be adopted by Bootstrap alike weighted procedure in nonlinear and nonGaussian formula. The simulation results show that firm's market value could be estimated purely and the relative variance is also evaluated with less noisy inflation. Thus, the distance to default with particle filter could be relatively properly evaluated.

\section{References}

[1] M. S. Arulapalam, S. Maskell, N. Gordon, and T. Clapp, "A Tutorial on Particle Filters for Online Nonlinear/Non-Gaussion Bayesian Tracking,"
IEEE Transactions on Signal Processing, Vol. 50, No. 2, pp. 174-188, 2002.

[2] F. Black, and M. Scholes, "The Pricing of Options and Corporate Liabilities,' Journal of Political Economy, Vol. 81, No. 3, pp. 637-654, 1973.

[3] P. P. Boyle, "Options: A Monte Carlo Approach,' Journal of Financial Economics, Vol. 4, pp. 323-338, 1977.

[4] A. W. Bulter, and L. Fauver, "Legal and Economic Determinants of Sovereign Credit Ratings,' Working Paper, 2005.

[5] P. Collin-Dufresne, R. S. Goldstein, and J. S. Martin, "The Determinants of Credit Spread Changes,' Journal of Finance, Vol. 56, No. 6, pp. 2177-2207, 2001.

[6] A. Doucet, S. Godsill, and C. Andrieu, "On Sequential Monte Carlo Sampling Methods for Bayesian Filtering," Statistics and Computing, Vol. 10, No. 3, pp. 197-208, 2000.

[7] J. C. Duan, and A. Fulop, "Estimating the Structural Credit Risk Model When Equity Prices are Contaminated by Trading Noises,", Working Paper, 2005.

[8] E. J. Elton, M. J. Gruber, D. Agrawal, and C. Mann, "Explaining the Rate Spread on Corporate Bonds,', Journal of Finance, Vol. 56, No. 1, pp. 247-277, 2001.

[9] N. J. Gordon, D. J. Salmond, and A. F. M. Smith, "Novel Approach to Nonlinear/Non-Gaussian Bayesian State Estimation,', IEE Proceedings- $F$, Vol. 40, No. 2, pp.107-113, 1993.

[10] D. J. Kisgen, "Credit Ratings and Capital Structure,'” Journal of Finance, forthcoming.

[11] M. T. Lin, J. L. Xhang, Q. Cheng, and R. Chen, "Independent Particle Filters,' Journal of the American Statistical Association, Vol. 100, No. 472, pp.1412-1421, 2005.

[12] R. C. Merton, "On the Pricing of Corporate Debt: The Risk Structure of Interest Rates," Journal of Finance, Vol. 29, No. 2, pp. 449-470, 1974.

[13] M. K. Pitt, and N. Shephard, "Filter via Simulation: Auxiliary Particle Filters,' Journal of the American Statistical Association, Vol. 94, No. 446, pp. 590-599, 1999.

[14] M. Vassalou, and Y. Xing, "Default Risk in Equity Returns,' Journal of Finance, Vol. 59, No. 2, pp.831-868, 2004.

[15] F. Yu, "Accounting Transparency and the Term Structure of Credit Spreads,' Journal of Financial Economics, Vol. 75, No. 1, pp. 53-84, 2005. 